\title{
BMJ Open Association between dairy intake and fracture in an Australian-based cohort of women: a prospective study
}

\author{
Hajara Aslam, ${ }^{1}$ Kara L Holloway-Kew, ${ }^{1}$ Mohammadreza Mohebbi, ${ }^{2}$ \\ Felice N Jacka, ${ }^{1,3,4}$ Julie A Pasco ${ }^{1,5,6,7}$
}

To cite: Aslam H, HollowayKew KL, Mohebbi M, et al. Association between dairy intake and fracture in an Australian-based cohort of women: a prospective study. BMJ Open 2019;9:e031594. doi:10.1136/ bmjopen-2019-031594

- Prepublication history and additional material for this paper are available online. To view please visit the journal (http:// dx.doi.org/10.1136/bmjopen2019-031594).

Received 14 May 2019 Revised 03 October 2019 Accepted 17 October 2019

Check for updates

(C) Author(s) (or their employer(s)) 2019. Re-use permitted under CC BY-NC. No commercial re-use. See rights and permissions. Published by BMJ.

For numbered affiliations see end of article.

Correspondence to

Hajara Aslam;

habdussa@deakin.edu.au

\begin{abstract}
Objective Given the inconsistent evidence on dairy consumption and risk of fracture, we assessed the association between milk/total dairy consumption and major osteoporotic fracture (MOF) in women from the Geelong Osteoporosis Study (GOS).
\end{abstract}

Methods Women aged $\geq 50$ years $(n=833$ ) were followed from baseline (1993-1997) to date of first fracture, death or 31 December 2017, whichever occurred first. Dairy consumption was assessed by self-report at baseline and the follow-up phases. MOFs (hip, forearm, clinical spine and proximal humerus) were confirmed radiologically. Multivariable-adjusted Cox proportional hazard models were used to determine associations between milk/total dairy (milk, cheese, yoghurt, ice cream) consumption and MOFs. Cross-sectional associations between milk/ total dairy consumption and serum high-sensitivity $C$ reactive protein (hsCRP), C-terminal telopeptide (CTx) and procollagen type $1 \mathrm{~N}$-terminal propeptide (P1NP) at baseline were investigated using multivariable linear regression.

Results During follow-up (11507 person-years), 206 women had an MOF. Consuming $>500 \mathrm{~mL} / \mathrm{d}$ of milk was not significantly associated with increased HR for MOF. Non-milk (1.56; $95 \% \mathrm{Cl} 0.99$ to 2.46) drinkers and consumption of $\geq 800 \mathrm{~g} / \mathrm{d}$ total dairy $(1.70 ; 95 \% \mathrm{Cl} 0.99$ to 2.93) had marginally higher HR for MOF compared with consuming $<250 \mathrm{~mL} / \mathrm{d}$ of milk and $200-399 \mathrm{~g} / \mathrm{d}$ of total dairy, respectively. Milk consumption was inversely associated with serum hsCRP and CTx, but total dairy consumption was not associated with these serum markers.

Conclusion Higher milk consumption did not increase the risk for MOF in older women. However, a trend for increased MOF was detected in zero milk and higher total dairy consuming women.

\section{INTRODUCTION}

Osteoporosis is a chronic multifactorial disease that is defined as low bone mass and impaired bone microarchitecture. ${ }^{12}$ The presence of osteoporosis substantially increases the risk of sustaining a fracture, especially at the hip, spine, forearm and proximal humerus, which are known as the major osteoporotic fracture (MOF) sites. However, the largest absolute number of fractures occurs

\section{Strengths and limitations of this study}

Although this study contained a modest sample size, it replicated the findings of previous studies

- Random sample selection from the general population is a strength of the study.

- The prospective study design strengthens the outcomes of the study despite methodological inconsistencies in capturing dietary data.

- As data for total dairy consumption were assessed at baseline only, we cannot account for dietary changes during follow-up and this limits the interpretation of the longitudinal analysis of the association between total dairy consumption and the risk for major osteoporotic fracture.

- The conclusions of this study cannot be generalised to a broader population as this study was focused on a cohort of women.

in people with a moderate deficit in bone density (osteopaenia). ${ }^{34}$ Falls risk, which can be affected by factors such as medication use, mobility level and environmental hazards, is also an important consideration, as most fractures are preceded by a fall..$^{5-8}$ Of the factors (eg, genetics, ${ }^{9}{ }^{10}$ age, ${ }^{11}{ }^{12}$ lifestyle habits, ${ }^{2}$ $\operatorname{sex}^{13}$ ) influencing fractures, nutrition plays a substantial role in the aetiology of osteoporosis. ${ }^{14} 15$ Adequate calcium and protein intakes are necessary in order to maintain skeletal integrity and strength. ${ }^{16}{ }^{17}$ Milk/ dairy products are key components in the western diet and contain a myriad of nutritional components (calcium, vitamins and proteins), and a majority of an individual's dietary calcium needs are fulfilled by intake of dairy products. ${ }^{18}{ }^{19}$ Additionally, milk/dairy products have been widely recommended to osteoporosis patients by clinicians and healthcare professionals considering the beneficial effects associated with dairy consumption. ${ }^{2021}$

However, data regarding milk consumption as a strategy for fracture prevention have shown inconclusive results. Findings from large Swedish cohorts reported that women 
who consumed three or more glasses of milk per day had higher risk for any fractures, while fermented dairy consumption was inversely associated with fractures. ${ }^{22}$ However, Feskanich $e t a l^{23}$ have shown in two large US cohorts that each serving of milk per day was associated with an $8 \%$ reduction of risk for hip fracture, whereas total dairy intake was associated with a $6 \%$ reduction of risk for hip fractures in men and women combined. Holvik et $a l^{24}$ found no association between increased milk intake and risk for hip fractures in Norwegian women and men. The most recently published meta-analysis (2018), which included 18 observational studies, showed that higher milk intakes were not associated with fractures in both sexes combined. ${ }^{25}$ However, it is worth noting that there was a large amount of heterogeneity between studies in terms of reporting milk/dairy intake, number of fractures, use of different confounders for adjustment and fracture ascertainment methods.

Although the overall evidence on increased milk intake appears supportive of reducing fractures, dissecting milk further to the molecular level demonstrates that milk contains compounds such as D-galactose (milk sugar) and A1-beta-casein (mutated protein variant) that may be detrimental to bone health. ${ }^{26-28}$ Preclinical studies show that these compounds are implicated in inflammation and oxidative stress pathways that can negatively impact bone metabolism. ${ }^{29}$ Moreover, Pasco et a ${ }^{31}$ previously indicated that increased milk intake is associated with depressive disorder, a condition that is comorbid with fractures. ${ }^{32} 33$ Therefore, we hypothesised that increased milk consumption may be associated with increased risk for MOF by triggering inflammation and oxidative stress.

Other milk-derived products such as yoghurt and cheese have a distinct biological profile to milk and may have a protective role in bone health due to the presence of probiotics, prebiotics and other bioactive compounds; these in turn have the potential to attenuate inflammation and oxidative stress. ${ }^{34}{ }^{35}$ Studies have assessed the effects of these products on bone separately to milk; however, the synergistic impact of dairy products (including milk, yoghurt, cheese, ice cream) with different molecular and biological profiles is poorly unravelled. Therefore, we aimed to assess the association between total dairy consumption and MOF in women. We also investigated potential mechanisms by which milk/total dairy may mediate the risk for MOF. For this purpose, the crosssectional association between milk/total dairy consumption and serum high-sensitivity $\mathrm{C}$ reactive protein (hsCRP), C-terminal telopeptide (CTx) and procollagen type $1 \mathrm{~N}$-terminal propeptide (P1NP) were examined at baseline.

\section{METHODS}

\section{Patient and public involvement}

Patients were not involved in the planning and design of the study.

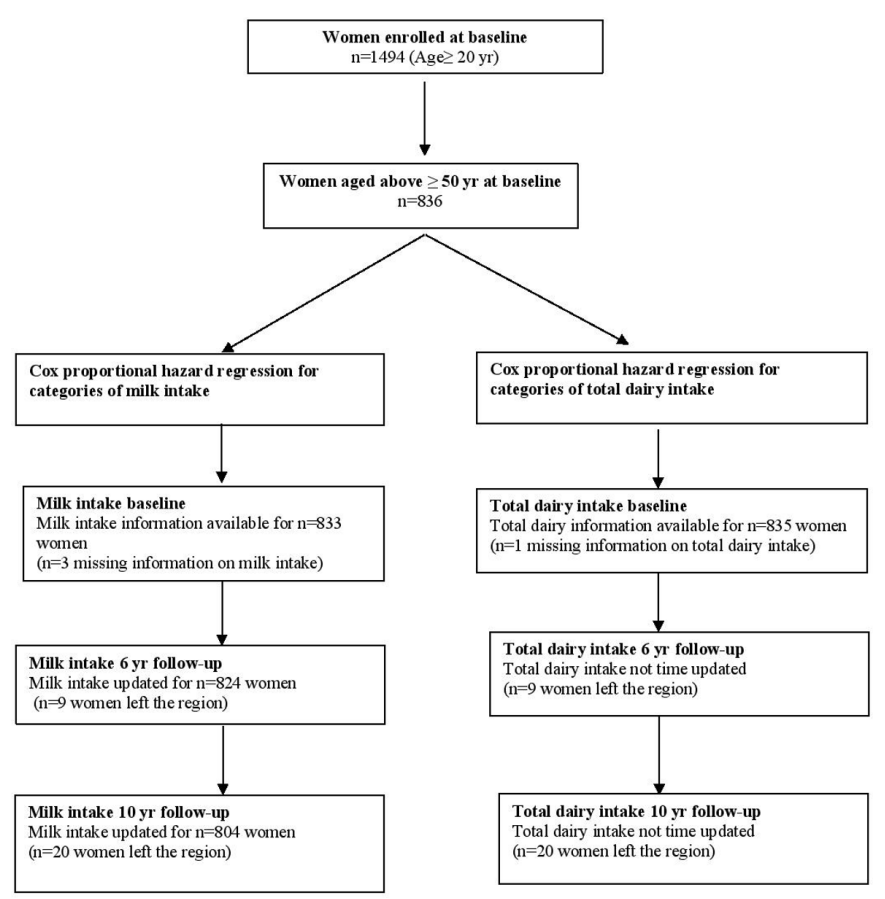

Figure 1 Participant flow chart. The figure represents the number of women at baseline, 6-year and 10-year follow-up waves and women left the region.

\section{Study population}

This study used data from the Geelong Osteoporosis Study (GOS), a large population-based cohort study based in south-eastern Australia. Inclusion criteria were: living in the Barwon Statistical Division (BSD) for $>6$ months and able to provide written informed consent. Women in the BSD were selected at random from the electoral roll during the years 1993-1997 to participate in the study. ${ }^{36}$ An age-stratified sample of 1494 women was enrolled in the study with a participation of $77.1 \%$. Subsequent assessments for these women commenced in 1995, 1998, 2000, 2002 and 2004, referred to as 2-year, 4-year, 6-year, 8 -year and 10-year follow-up phases. The cohort profile is explained elsewhere.$^{36}$ For the purposes of the analysis, women only $\geq 50$ years at baseline were considered. Of the 836 women aged $\geq 50$ years, 833 women were included in the analysis after excluding records with missing information on milk intake (figure 1). Study participants provided written informed consent. The study was approved by the Human Research Ethics Committee at Barwon Health.

\section{Outcome measures}

Postbaseline incident fractures were identified using a method that have been validated for fracture ascertainment in the region. Radiological reports (X-ray) of fractures from all radiological centres in the region were scrutinised to identify and confirm fractures. ${ }^{37} 38$ Trained research personnel examined each record individually and determined the most appropriate international code of diseases version 9 (ICD-9) codes for fracture site, as well as level of trauma. ${ }^{39}$ MOFs were defined as fractures at the hip, forearm, clinical spine and proximal 
humerus, according to the Fracture Risk Assessment Tool (FRAX) developed by the University of Sheffield for clinical use. ${ }^{40}$ Pathological and high trauma fractures were excluded. Information on death was collected from the National Deaths Index (Australian Institute for Health and Welfare).

\section{Dairy consumption and diet}

Information on dairy was available at baseline, 6-year and 10-year follow-up. At baseline and 6-year follow-up, dietary information was documented by a self-reported questionnaire that contained questions on 35 foods and beverages on average. Participants were asked questions about the habitual/type of (all forms, eg, milk used in cooking, baking and in coffee) milk consumed (whole, reduced fat, calcium fortified, soy, goat's milk, butter milk and evaporated) and the quantity consumed each day. In the questionnaire, it was stated that one cup of milk is considered equivalent to $250 \mathrm{~mL}$. Therefore, participants chose the type and quantity of milk consumed from any predetermined milk categories and only cow's milk was considered (none, $<125 \mathrm{~mL}$ ( $<1 / 2 \mathrm{cup}$ ), $125-249 \mathrm{~mL}$ (1/2 to $<1$ cup), $250-499 \mathrm{~mL}$ ( 1 to $<2$ cups), $500-999 \mathrm{~mL}$ ( 2 to $<4$ cups), $\geq 1000 \mathrm{~mL}$ ( $\geq 4$ cups) per day). The lowest response categories, $<125 \mathrm{~mL} / \mathrm{d}, 125-249 \mathrm{~mL} / \mathrm{d}$ were collapsed into one category indicating ' $<250 \mathrm{~mL} / \mathrm{d}$ ' and the highest response categories, $500-999 \mathrm{~mL} / \mathrm{d}, \geq 1000 \mathrm{~mL}$ were combined into one category indicating ' $>500 \mathrm{~mL} / \mathrm{d}$ '; this was due to low proportions responding to the lower and higher categories and for the compatibility with the 10-year follow-up dietary data. The second lowest category was chosen as reference for milk consumption as this category benefits robustness due to higher number of participants within the category.

Information on other dairy products such as cheese, yoghurt (all forms, eg, cheese and yoghurt used in cooking, baking) and ice cream consumption were also documented using this self-reported questionnaire. Participants were specifically asked about different types of cheese they consumed on a weekly basis including hard cheese, for example, cheddar, tasty (servings/ week; 1 serving=16g); soft cheese, for example, cream, cottage (servings/week; 1 serving=20 g); and fruche (servings/week; 1 serving=100 g). Fruche is a form of soft cheese (fromage frais) and thus was categorised as cheese. Total cheese consumption was converted to grams consumed per day $(\mathrm{g} / \mathrm{d})$. Yoghurt (servings/week; 1 serving=200 g) and ice cream consumption (servings/ week; 1 serving $=27 \mathrm{~g}$ ) were reported as servings per week, and this was converted to grams consumed per day. Daily total dairy consumption was calculated at baseline by combining values for cow's milk, all forms of cheese, yoghurt and ice cream consumed and was expressed in grams consumed per day. The clustered nature of total dairy distribution made it unfeasible to consider it as a continuous variable for analytical purpose, and as such it was treated as categorical variable in the analysis and categorised as $<200,200-399,400-700$ and $\geq 800 \mathrm{~g} / \mathrm{d}$. The second lowest category was chosen as reference for total dairy because it was the largest group.

At 10-year follow-up, information on milk/dairy consumption was collected using a validated food frequency questionnaire. The Cancer Council Victoria Dietary Questionnaire captures information on 74 foods and 6 alcoholic beverages over the previous 12 months and is validated for assessing habitual dietary intake in Australian women. ${ }^{41}$ Participants were queried on their usual type (none, full cream, reduced fat, skim and soy milk) and quantity of milk consumed on a daily basis. Participants were advised that 1 cup of milk is equivalent to $250 \mathrm{~mL}$ of milk. Furthermore, participants indicated their daily milk intake by selecting from predetermined categories of milk intakes and only cow's milk was considered (none, $<250 \mathrm{~mL}$ ( $<1 \mathrm{cup}$ ), $250-499 \mathrm{~mL}$ ( 1 to $<2$ cups), $500-750 \mathrm{~mL}$ ( 2 to 3 cups) and $>750 \mathrm{~mL}$ ( $>3$ cups) per day). The highest response categories, $500-750 \mathrm{~mL} / \mathrm{d}$, $>750 \mathrm{~mL} / \mathrm{d}$ were combined as to one category indicating ' $>500 \mathrm{~mL} / \mathrm{d}$ '; this was due to low proportions responding to the higher categories. This questionnaire also captured information on cheese, yoghurt and ice cream intake of participants.

A separate calcium-specific dietary questionnaire was used to capture information on dietary calcium intake. This questionnaire included information on a range of common calcium-dense food sources, which allowed calculation of dietary calcium intakes in $\mathrm{mg}$ per day $(\mathrm{mg} / \mathrm{d})$ and validated against 4-day weighed food intakes. ${ }^{19}$ Dietary calcium intake was categorised into two strata $(<1000 \mathrm{mg} / \mathrm{d}, \geq 1000 \mathrm{mg} / \mathrm{d})$.

\section{Other information and potential confounders}

All measurements were assessed at the baseline visit. Weight and height were recorded to the nearest $0.1 \mathrm{~kg}$ and $0.1 \mathrm{~cm}$, respectively, and body mass index (BMI) calculated as weight $/$ height $^{2}\left(\mathrm{~kg} / \mathrm{m}^{2}\right)$. Dual-energy X-ray absorptiometry (Lunar DPX-L; Lunar, Madison, Wisconsin, USA) was performed to evaluate bone mineral density (BMD; $\mathrm{g} / \mathrm{cm}^{2}$ ) at the femoral neck, and whole-body fat $(\mathrm{kg})$ and 'lean' mass $(\mathrm{kg})$ that represents the water and protein content in muscle, skin, connective tissue and lean component in adipose tissue.

Self-report questionnaires were used to obtain information on mobility, physical activity levels, smoking status, medications, prior falls and fractures. Participants were asked to select their mobility level from predetermined 7-point scale (very active, active, sedentary, limited, inactive, chair or bed ridden, bedfast-examples were given in the questionnaire to assist the participant to choose the most suitable option). These categories were further condensed to two groups, highly active and less active, for the purpose of this analysis. Physical activity level was also assessed from questions regarding work/home and recreational/sports, on a 3-point scale, which provided options for participants to select from moderate, hard and very hard. Participants were also asked to enter the time spent on each activity level on a weekly basis. 
Information on current smoking status was categorised as smoking or non-smoking. Use of medications that positively or negatively influences bone included bisphosphonates, anabolic therapies, hormonal replacement therapies (HTs) and oral glucocorticoids. Participants were asked to list the use of supplements, and this information was used to assess the calcium and vitamin D supplementation usage. Use of supplementary calcium and vitamin $\mathrm{D}$ was documented at baseline, 6-year and 10-year follow-up.

The definition of falls (when you suddenly find yourself on the ground, without intending to get there, after you were in a lying, sitting or standing position) was explicit in the questionnaire and asked participants whether or not they experienced a similar scenario over the past 12 months. Information regarding previous fractures and cancer diagnoses was also captured by self-reported questionnaires. An automated device (Takeda Medical UA-751) was used to measure blood pressure in a sitting position. Women were considered hypertensive if they had a systolic blood pressure over $140 \mathrm{~mm} \mathrm{Hg}$ and/or a diastolic pressure above $90 \mathrm{~mm} \mathrm{Hg}$ and/or use of antihypertensive medication in the presence of self-reported hypertension. Women were identified as having diabetes if they had a fasting plasma glucose $\geq 7.0 \mathrm{mmol} / \mathrm{L}$ (126 mg/ $\mathrm{dL}$ ), self-reported diabetes and/or use of antihyperglycaemic agents.

Information pertinent to educational qualifications were gathered on a 7-point scale: never attended school, primary school, some secondary school, completed secondary school, post-secondary qualifications, university or other tertiary qualifications and cannot remember. These categories were compressed to education received for less than 12 years or more than 12 years for the purpose of this analysis. Information on marital status was dichotomised as living alone or living with a partner. The socioeconomic status of the cohort participants was measured by the Index of Relative Socioeconomic Disadvantage (IRSD), an area-based index that measures relative disadvantage of socioeconomic status. This tool imputes a span of information on economic and social conditions of people and household within an area and is represented in quintiles. The most disadvantaged category is indexed by quintile $1 .^{42}$

\section{Biomarkers}

At baseline, venous blood was collected after an overnight fast and stored at $-80^{\circ} \mathrm{C}$ until batch analysis. Markers of bone turnover, serum CTx, a marker of bone resorption, and serum P1NP, a marker of bone formation, were analysed from the blood samples. In addition, serum hsCRP, a marker of systemic inflammation, was determined from the blood samples. Serum hsCRP was measured by the Roche immunoturbidimetric 'CRP' and 'C-reactive protein (latex) high sensitivity methods'. Details of these analytical methods have been described elsewhere. $^{43} 44$

\section{Statistical analysis}

Characteristics of participants were described by mean $( \pm \mathrm{SD})$ or median $(\mathrm{IQR})$ or relative frequencies $(\%)$ stratified by milk consumption categories (no milk, $<250 \mathrm{~mL} / \mathrm{d}$, $250-500 \mathrm{~mL} / \mathrm{d},>500 \mathrm{~mL} / \mathrm{d}$ ). Participant characteristics across categories of milk consumption were compared using one-way ANOVA or Kruskal-Wallis $\mathrm{H}$ test for continuous data and $\chi^{2}$ test (or Fisher's exact test) for categorical data. The null hypothesis was rejected at an $\alpha$ level of 0.05 , and a post hoc multiple comparison was performed using Bonferroni corrections. Additionally, participant characteristics were described based on total dairy categories $(<200 \mathrm{~g} / \mathrm{d}, 200-399 \mathrm{~g} / \mathrm{d}, 400-799 \mathrm{~g} / \mathrm{d}, \geq 800 \mathrm{~g} / \mathrm{d})$ (online supplementary table 1 ).

Cohort participants were followed from their baseline appointment to date of first fracture, death or 31 December 2017. Cox proportional hazard regression was used to estimate age-adjusted HR and their $95 \%$ CIs for categories of milk consumption (no milk, $<250 \mathrm{~mL} / \mathrm{d}$, $250-500 \mathrm{~mL} / \mathrm{d},>500 \mathrm{~mL} / \mathrm{d})$.

Covariates (BMD, BMI, smoking, alcohol consumption, prebaseline fractures incidents, diabetes, IRSD, education, mobility, medications that influence bone metabolism, calcium and vitamin D supplements) were assessed in bivariate Cox regression analysis to determine their impacts on the association between milk/total dairy consumption and fractures. The covariates that impacted the HR when added or removed (considering the statistical significance and change of HR in the exposure of interest) from the model were included in the final Cox regression model. In addition, when deciding on the confounders, the potential of the covariate to be associated with both the exposure and the outcome was also considered. The final model consisted of age, oral glucocorticoid use, HT use and prebaseline fractures as confounders. Information on milk consumption, oral glucocorticoid use and HT use were time updated at the 6 -year and 10-year follow-up. Age was time updated in all follow-up waves. Information on prebaseline fractures were not time updated and kept constant for the analysis. We also performed a multivariable-adjusted sensitivity analysis using baseline milk values only.

In addition, a Cox proportional hazard regression was also used to estimate the age-adjusted HR and their 95\% CIs for total dairy consumption categories $(<200 \mathrm{~g} / \mathrm{d}$, 200-399 g/d, 400-799 g/d, $\geq 800 \mathrm{~g} / \mathrm{d}$ ). Selection of potential confounders was performed according to the aforementioned method. The final model consisted of age, oral glucocorticoid use, HT use and prior fractures as confounders. For this analysis, total dairy consumption was not time updated due to the inconsistent dietary tools used in capturing information on dairy products during the follow-up waves; therefore, baseline-only values for total dairy consumption were used in the analysis. Oral glucocorticoid use and HT use were time updated at the 6-year and 10-year follow-up; age was time updated at all follow-up waves; prebaseline fractures were not time updated and kept constant for the analysis. 
The proportional hazard assumptions were confirmed graphically by $\log (-\log ($ survival $))$ plots for both daily milk and total dairy consumption. Time to first fracture (survival) curves were illustrated using Kaplan-Meier estimator of the survival function using product limit estimator.

The cross-sectional associations between milk/total dairy consumption and serum markers of inflammation (hsCRP) and bone turnover (CTx, P1NP) were assessed using multivariable linear regression models at baseline with potential confounders. Women who had missing information $(n=45)$ on inflammatory and bone turnover markers were excluded from the analysis. Serum markers of inflammation and bone turnover were log transformed due to the skewed nature of data. For all analyses, STATA V.15 and SPSS V.25 were used.

\section{RESULTS}

Descriptive characteristics of the cohort stratified by milk consumption categories are presented in table 1. Of 833 women, $8.4 \%(\mathrm{n}=70)$ did not consume milk and $47.2 \%$ $(\mathrm{n}=393), 34.3 \%(\mathrm{n}=286)$ and $10.0 \%(\mathrm{n}=84)$ consumed $<250 \mathrm{~mL} / \mathrm{d}, \quad 250-500 \mathrm{~mL} / \mathrm{d}$ and $>500 \mathrm{~mL} / \mathrm{d}$ of milk, respectively. There was no difference observed in women's median age among the four milk consumption categories. Women who consumed $>500 \mathrm{~mL} / \mathrm{d}$ of milk reported the highest cheese intake. The group that consumed $<250 \mathrm{~mL} / \mathrm{d}$ of milk had the highest proportion of women reporting $<1000 \mathrm{mg} / \mathrm{d}$ of dietary calcium intake. On the contrary, the group that consumed $>500 \mathrm{~mL} / \mathrm{d}$ of milk had the highest proportion of women reporting $\geq 1000 \mathrm{mg} / \mathrm{d}$ of dietary calcium intake (table 1 ). The proportion of women consuming supplementary calcium and vitamin $\mathrm{D}$ was high among the non-milk consumers. There were no differences detected for other parameters across the four milk consuming groups.

During 11507 person years of follow-up, 206 women sustained an MOF (spine $=96$; humerus $=14$; wrist $=51$; hip $=45$ ) and 503 women died. Women who consumed no milk reported the highest fracture rate (table 2) and the crude fracture survival probability curve also showed that women who consumed no milk had the lowest survival probability for fractures (figure 2). Concordantly, women who reported no milk consumption showed marginally significant higher age-adjusted (1.54, 95\% CI 0.98 to 2.44 , $\mathrm{p}=0.06)$ and multivariable-adjusted $(1.56,95 \% \mathrm{CI} 0.99$ to 2.46, $\mathrm{p}=0.06$ ) HR for MOF compared with women who consumed $<250 \mathrm{~mL} / \mathrm{d}$ of milk. The unadjusted $(1.28$, $95 \%$ CI 0.84 to $1.96, \mathrm{p}=0.25)$, age-adjusted $(1.23,95 \% \mathrm{CI}$ 0.80 to $1.88, \mathrm{p}=0.34)$ and multivariable-adjusted (1.15, $95 \%$ CI 0.75 to $1.75, \mathrm{p}=0.53$ ) HR for MOF were not significantly higher in women who consumed $>500 \mathrm{~mL} / \mathrm{d}$ of milk compared with women who consumed $<250 \mathrm{~mL} / \mathrm{d}$ of milk. The multivariable-adjusted sensitivity analysis that was performed using baseline milk values only resulted in a non-significant higher HR for non-milk consumers (HR: 1.53 ; CI 0.96 to $2.44 ; \mathrm{p}=0.07$ ) and $>500 \mathrm{~mL} / \mathrm{d}$ of milk consumers (HR: 1.13; CI 0.74 to 1.72; $\mathrm{p}=0.58$ ) compared with consuming $<250 \mathrm{~mL} / \mathrm{d}$ of milk.

When total dairy consumption was considered, women who consumed $\geq 800 \mathrm{~g} / \mathrm{d}$ demonstrated the highest fracture rate (table 3 ). This was also confirmed by the crude fracture survival probability curve, which indicated the lowest survival probability for fractures in women who consumed $\geq 800 \mathrm{~g} / \mathrm{d}$ total dairy (figure 3). Consistently, women who consumed $\geq 800 \mathrm{~g} / \mathrm{d}$ total dairy showed higher age-adjusted (2.01, 95\% CI 1.88 to 3.44, $\mathrm{p}=0.01)$ and multivariable-adjusted $(1.70,95 \%$ CI 0.99 to 2.93, $\mathrm{p}=0.05$ ) HR for MOF compared with women who consumed 200-399g/d of total dairy (table 3).

An inverse association was observed between milk consumption and serum markers of inflammation (hsCRP) and serum markers of bone resorption (CTx); women who consumed $>500 \mathrm{~mL} / \mathrm{d}$ of milk had the lowest concentrations of serum hsCRP $(-0.45 ; 95 \%$ CI -0.82 to $0.07 ; \mathrm{p}=0.02)$ and serum CTx $(-0.25 ; 95 \%$ CI -0.48 to $0.02 ; \mathrm{p}=0.03$ ) (table 4 ). No association was found between milk consumption and serum marker of bone formation (P1NP). Moreover, there was no association found between total dairy consumption categories and serum hsCRP, CTx and P1NP (table 4).

\section{DISCUSSION}

In our study of older Australian women, we detected no significant association between higher milk consumption $(>500 \mathrm{~mL} / \mathrm{d})$ and increased risk for MOF. However, we found that zero milk and higher total dairy $(\geq 800 \mathrm{~g} / \mathrm{d})$ consumptions had marginally higher risk for MOF.

Acquiring the daily recommend calcium through diet/ supplements is considered the easiest and safest lifestyle modification that could be achieved as a part of prevention and management of osteoporosis. ${ }^{45}$ Milk/dairy products are considered the ideal source of calcium that consumed in recommended quantities may approximately satisfy the daily calcium requirements. ${ }^{46}{ }^{47}$ In general, $1200 \mathrm{mg} / \mathrm{d}$ of calcium is recommended for women aged $>50$ years ${ }^{48}$ and potentially four serves of milk (one serve $=250 \mathrm{~mL}=300 \mathrm{mg}$ of calcium) can cover this need. Our study results were in support of this, suggesting that not consuming milk may increase the risk of MOF.

However, some components in milk such as D-galactose $^{49}$ and A1-beta-casein ${ }^{50}$ are believed to possibly mediate the unfavourable consequences associated with milk consumption. D-galactose has proven to be involved in the ageing process in mice, which encompassed series of events such as oxidative stress and chronic inflammation. ${ }^{26}$ Besides, existing epidemiological data show that some negative health consequences (ischaemic heart disease and type 1 diabetes) associated with milk consumption may be due to the A1-beta-casein fraction in milk. ${ }^{51-55}$ However, robust evidence from clinical trials is lacking to confirm causality.

The Swedish cohort study speculated that increased milk intake may be deleterious to bone due to the 
Open access

Table 1 Baseline characteristics of participants stratified by milk consumption categories ${ }^{\star}$

\begin{tabular}{|c|c|c|c|c|}
\hline & No milk & $<250 \mathrm{~mL} / \mathrm{d}$ & $250-500 \mathrm{~mL} / \mathrm{d}$ & $>500 \mathrm{~mL} / \mathrm{d}$ \\
\hline Number of women & 70 & 393 & 286 & 84 \\
\hline Age at entry, years & $68.2(58.2-77.6)$ & $69.1(59.2-80.3)$ & $71.4(60.5-80.4)$ & $71.7(64.2-80.4)$ \\
\hline Body mass index, $\mathrm{kg} / \mathrm{m}^{2}$ & $25.1(22.1-28.6)$ & $26.8(24.1-30.3)$ & $25.9(23.5-29.9)$ & $25.3(23.2-28.9)$ \\
\hline Yoghurt, g/d & $0.0(0.0-57.1)$ & $0.0(0.0-57.1)$ & $3.6(0.0-57.1)$ & $0.0(0.0-85.7)$ \\
\hline Cheese, $g / d$ & $9.1(3.4-22.9)$ & $9.1(4.6-16.0)$ & $11.0(4.6-22.9)$ & $13.7(6.9-25.1) \dagger$ \\
\hline Ice cream, g/d & $0.0(0.0-11.6)$ & $0.0(0.0-7.7)$ & $0.0(0.0-7.7)$ & $0.0(0.0-11.6)$ \\
\hline Bone mineral density, $\mathrm{g} / \mathrm{cm}^{2}$ & $0.792 \pm 0.163$ & $0.830 \pm 0.156$ & $0.832 \pm 0.146$ & $0.808 \pm 0.161$ \\
\hline Whole body fat, kg & $24.1(18.9-32.2)$ & $27.6(20.7-34.0)$ & $25.7(20.5-32.6)$ & $24.6(19.5-29.0)$ \\
\hline Lean mass, $\mathrm{kg}$ & $36.3 \pm 4.8$ & $37.3 \pm 4.7$ & $37.3 \pm 4.6$ & $36.9 \pm 4.1$ \\
\hline \multicolumn{5}{|l|}{ Dietary calcium, n (\%) } \\
\hline$<1000 \mathrm{mg} / \mathrm{d}$ & $65(93)$ & $386(98) \dagger$ & $246(86)$ & $1(1)$ \\
\hline$\geq 1000 \mathrm{mg} / \mathrm{d}$ & $5(7)$ & $4(1)$ & 39 (13) & $82(98) \dagger$ \\
\hline Falls in the past, $\mathrm{n}(\%)$ & $14(20)$ & $73(19)$ & $63(20)$ & $21(25)$ \\
\hline Prebaseline fractures, n (\%) & $24(34)$ & $146(37)$ & $93(33)$ & $39(46)$ \\
\hline Incident cancer, n (\%) & $7(10)$ & $58(15)$ & $37(13)$ & $14(17)$ \\
\hline Diabetes, n (\%) & $6(9)$ & $30(8)$ & $22(8)$ & $11(13)$ \\
\hline Hypertension, n (\%) & $47(67)$ & $242(62)$ & $172(60)$ & $51(61)$ \\
\hline \multicolumn{5}{|l|}{ Smoking, n (\%) } \\
\hline Smokers & $64(91)$ & $348(89)$ & $267(93)$ & $78(93)$ \\
\hline Non-smokers & $6(9)$ & $45(11)$ & $19(7)$ & $6(7)$ \\
\hline \multicolumn{5}{|l|}{ Mobility, n (\%) } \\
\hline Highly active & $38(54)$ & $192(49)$ & $142(50)$ & $43(51)$ \\
\hline Less active & $32(46)$ & $201(51)$ & $144(50)$ & $41(49)$ \\
\hline Supplemental calcium, n (\%) & $16(22) \dagger$ & $49(12)$ & $34(12)$ & $17(20)$ \\
\hline Supplemental vitamin D, n (\%) & $15(21) \dagger$ & $45(11)$ & $25(9)$ & $15(18)$ \\
\hline Bisphosphonates, n (\%) & $1(1)$ & $1(0)$ & $6(2)$ & $0(0)$ \\
\hline Anabolic therapies, $\mathrm{n}(\%)$ & $0(0)$ & $0(0)$ & $0(0)$ & $0(0)$ \\
\hline HT, n (\%) & $10(14)$ & $70(18)$ & $38(13)$ & $8(10)$ \\
\hline Oral glucocorticoids, n (\%) & $2(3)$ & $9(3)$ & $5(2)$ & $6(7)$ \\
\hline \multicolumn{5}{|l|}{ IRSD n (\%)/quintile } \\
\hline 1 & $11(15)$ & $81(21)$ & $48(17)$ & $15(18)$ \\
\hline 2 & $18(26)$ & $81(21)$ & $63(22)$ & $19(24)$ \\
\hline 3 & $16(23)$ & $97(25)$ & $58(20)$ & $20(24)$ \\
\hline 4 & $12(17)$ & $58(14)$ & $55(19)$ & $14(14)$ \\
\hline 5 & $13(19)$ & $76(19)$ & $62(22)$ & $16(19)$ \\
\hline \multicolumn{5}{|l|}{ Education, n (\%) } \\
\hline$<12$ years & $63(90)$ & $342(87)$ & $248(87)$ & $69(82)$ \\
\hline$\geq 12$ years & $7(10)$ & $46(11)$ & $37(13)$ & $14(17)$ \\
\hline \multicolumn{5}{|l|}{ Marital status, n (\%) } \\
\hline Living with partner & $28(40)$ & $162(41)$ & $137(48)$ & $43(51)$ \\
\hline Living alone & $42(60)$ & $231(59)$ & $149(52)$ & $41(49)$ \\
\hline
\end{tabular}

The most disadvantaged category in IRSD is indexed by quintile 1.

${ }^{*}$ Data reported as mean $\pm \mathrm{SD}$, median (IQR) or $\mathrm{n}(\%)$; milk comprises skim, low fat, full fat with a serving size of $1 \mathrm{cup}=250 \mathrm{~mL}$.

$\dagger \mathrm{P}<0.01$ Bonferroni corrected.

HT, hormonal replacement therapy; IRSD, Index of Relative Socioeconomic Disadvantage. 
Table 2 Incident fracture rates (n/1000), unadjusted, age-adjusted and multivariable-adjusted HR for MOF in different milk consumption categories with their $95 \% \mathrm{Cl}^{*}$

\section{Categories of milk consumption*}

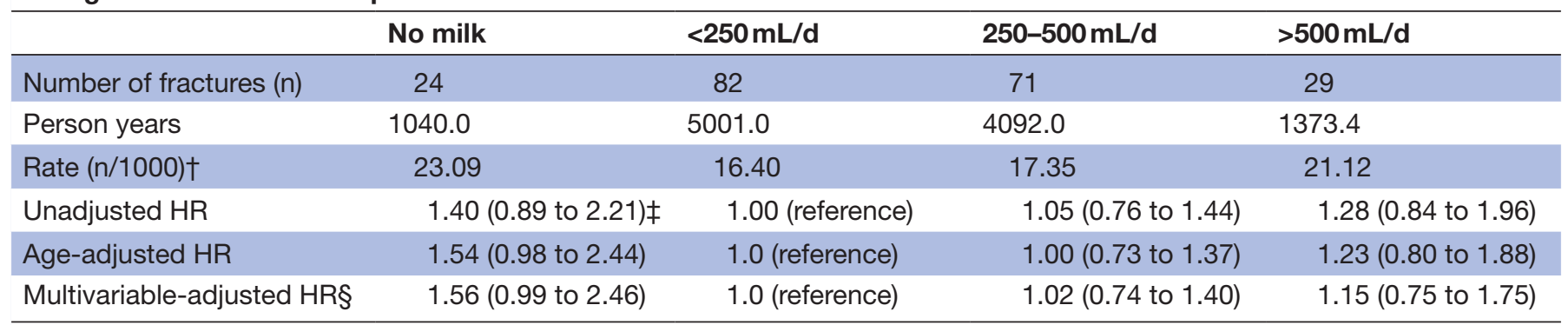

*Milk comprises skim, low fat, full fat with a serving size of $1 \mathrm{cup}=250 \mathrm{~mL}$ (time updated at 6 -year and 10-year follow-up waves). †Fracture rates: number of cases per 1000 person years at risk. $\ddagger 95 \% \mathrm{Cl}$ in parentheses (all such values).

§Adjusted for oral glucocorticoids, HT (time updated at 6-year, 10-year follow-up waves), age (time updated at all follow-up waves) and prebaseline fractures (baseline values).

HT, hormonal replacement therapy;MOF, major osteoporotic fracture (fractures in hip, forearm, clinical spine and proximal humerus).

D-galactose content in milk and showed that women who consumed more than three glasses of milk compared with one glass of milk per day had higher risk for any fractures and mortality. ${ }^{22}$ Additionally, a positive correlation between milk consumption and both oxidative stress marker in urine (8-iso-PGF2 $\alpha$ ) and inflammatory marker in serum (interleukin 6) were detected in the Swedish cohort. ${ }^{22}$ Hence, those findings offered support to the hypothesis that increased milk intakes are deleterious to bone and this may be mediated through D-galactose in milk. ${ }^{22}$ However, many other studies ${ }^{2425} 56$ including our study did not find any evidence to show that increased milk consumption is associated with fractures. We also assessed the association between total dairy consumption (milk, cheese, yoghurt and ice cream) and MOF. Here, we found that women who consumed $\geq 800 \mathrm{~g} / \mathrm{d}$ of total dairy showed higher risk for MOF compared with women consuming moderate levels.

This study also attempted to assess the underpinning mechanisms by which higher milk/total dairy products may instigate fractures. It was expected that increased milk/total dairy intakes may augment systemic inflammation, thereby negatively influence bone metabolism and increase bone fragility and risk for fractures. ${ }^{44} 57$

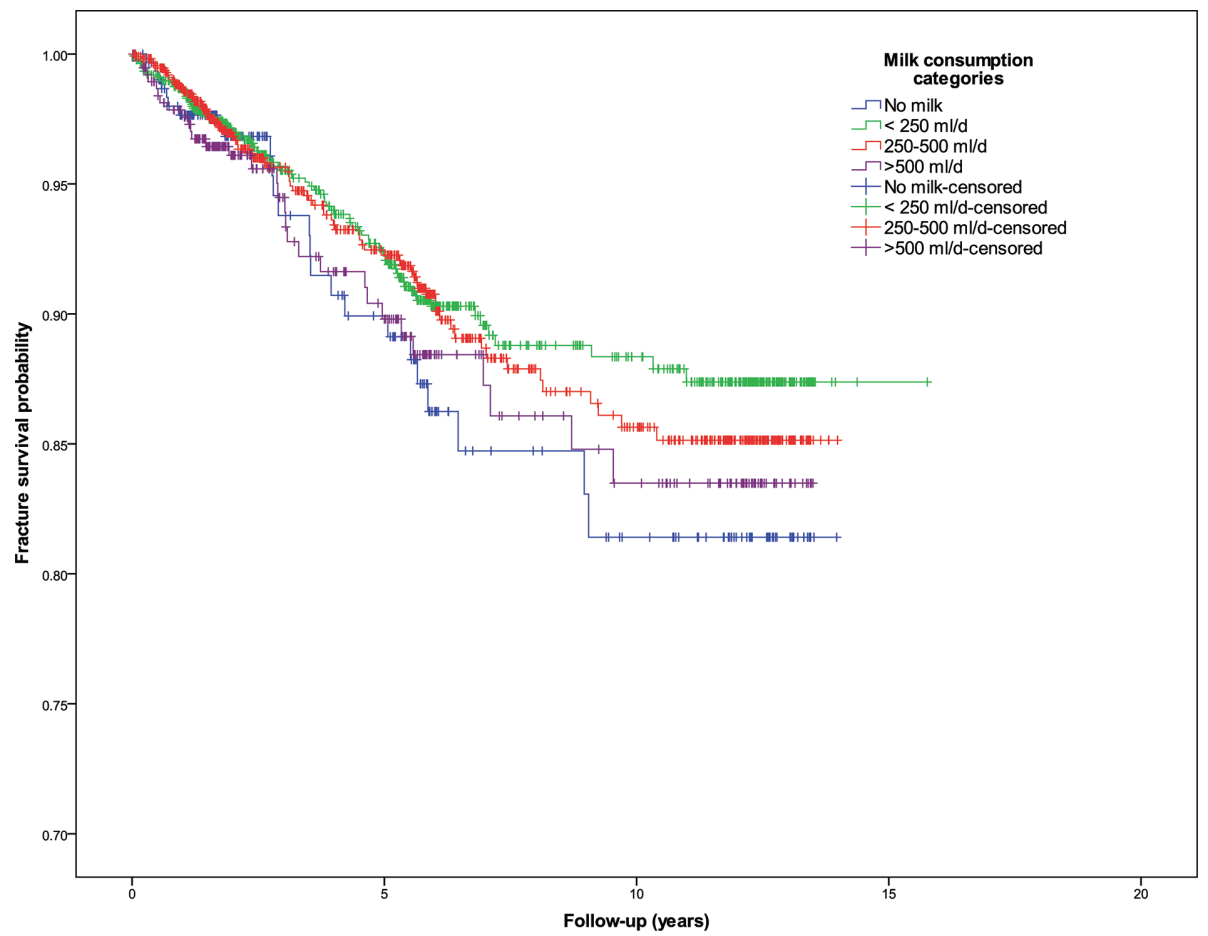

Figure 2 Kaplan-Meier survival plot for fractures in different milk consumption groups of women. The four curves represent fracture survival probability in different milk consumption groups (crude data). The lowest fracture survival probability is shown by the group consuming no milk. 
Table 3 Incident fracture rates (n/1000), unadjusted, age-adjusted and multivariable-adjusted HR for MOF in different total dairy products consumption categories with their $95 \% \mathrm{Cl} \dagger$

\section{Categories of total dairy consumption $\uparrow$}

\begin{tabular}{lcccc}
\hline & $\mathbf{2 0 0} \mathbf{g} / \mathbf{d}$ & $\mathbf{2 0 0 - 3 9 9} \mathbf{~} / \mathbf{d}$ & $\mathbf{4 0 0 - 7 9 9} \mathbf{~} / \mathbf{d}$ & $\mathbf{2 8 0 0} \mathbf{g} / \mathbf{d}$ \\
\hline Fractures & 61 & 66 & 62 & 17 \\
Person years & 3125.0 & 4362.1 & 3492.1 & 528.1 \\
Rate (per 1000)‡ & 19.52 & 15.13 & 17.75 & 32.19 \\
Unadjusted HR & $1.30(0.91$ to 1.83$) \S$ & 1.00 (reference) & $1.18(0.84$ to 1.68$)$ & $2.10(1.23$ to 3.58$)$ \\
Age-adjusted HR & $1.42(1.00$ to 2.01$)$ & 1.00 (reference) & $1.34(0.94$ to 1.90$)$ & $2.01(1.18 \text { to } 3.44)^{*}$ \\
Multivariable-adjusted HRף & $1.40(0.98$ to 1.97$)$ & 1.00 (reference) & $1.35(0.95$ to 1.91$)$ & $1.70(0.99$ to 2.93$)$ \\
\hline
\end{tabular}

${ }^{*} \mathrm{P}<0.05$.

†Total dairy includes milk, cheese, yoghurt and ice cream.

‡Fracture rates: number of cases per 1000 person years at risk.

$\S 95 \% \mathrm{Cl}$ in parentheses (all such values).

ПAdjusted for oral glucocorticoids, HT (time updated at 6-year, 10-year follow-up waves), age (time updated at all follow-up waves) prebaseline fractures (baseline values).

HT, hormonal replacement therapy;MOF, major osteoporotic fracture (fractures in hip, forearm, clinical spine and proximal humerus).

Serum hsCRP is deemed a sensitive marker of systematic inflammation and higher concentration of serum hsCRP has been detected in inflammatory diseases and also associated with fractures. ${ }^{58} 59$ Therefore, the crosssectional association between milk/total dairy categories and serum marker of inflammation (hsCRP) was tested. The lowest serum hsCRP concentration was detected in women who consumed $>500 \mathrm{~mL} / \mathrm{d}$ of milk. Our findings did not support our hypothesis, and they were corroborated by other literature that showed decreased CRP levels with increased milk/dairy intake. ${ }^{60}$ Also, we did not find any association between total dairy consumption and serum hsCRP. We also assessed whether there is an association between milk/total dairy consumption categories and markers of bone turnover. There were no clear patterns of associations found between milk consumption and serum marker of bone formation (P1NP). But women consuming $>500 \mathrm{~mL} / \mathrm{d}$ of milk had the lowest concentrations of serum marker of bone resorption $(\mathrm{CTx})$. Moreover, there was no association detected between total dairy consumption and serum CTx and P1NP.

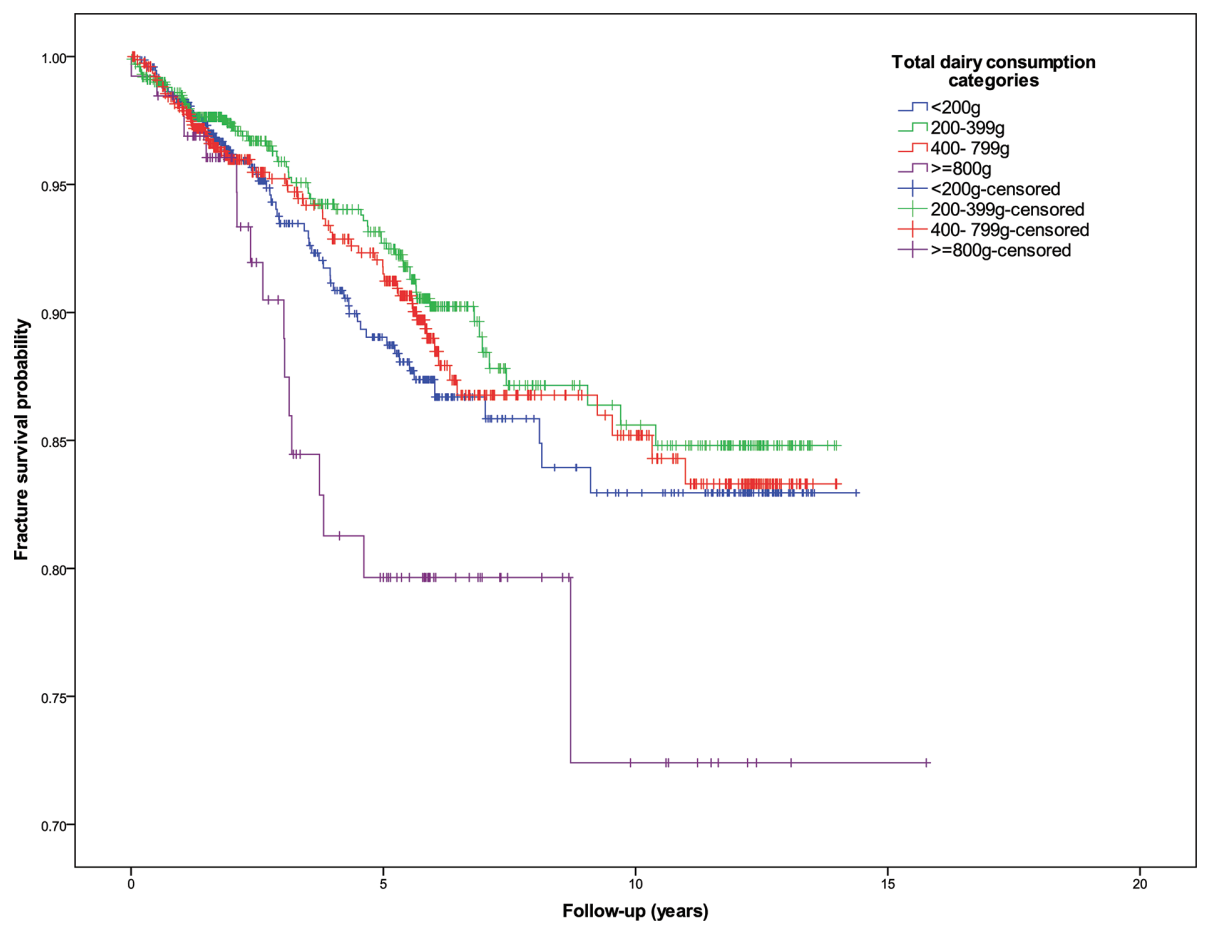

Figure 3 Kaplan-Meier survival plot for fractures in different total dairy consumption groups of women. The four curves represent fracture survival probability in different total dairy consumption groups (crude data). The lowest fracture survival probability is shown by the group consuming $\geq 800 \mathrm{~g} / \mathrm{d}$ total dairy. 
Table 4 Association between milk/total dairy consumption categories and serum markers of systemic inflammation and bone turnover with their $95 \% \mathrm{Cl} \dagger$

\begin{tabular}{|c|c|c|c|c|c|c|}
\hline & \multicolumn{2}{|c|}{ hsCRP (mg/L)‡ } & \multicolumn{2}{|l|}{ CTx (ng/L)§ } & \multicolumn{2}{|c|}{ P1NP $(\mu \mathrm{g} / \mathrm{L}) \eta$} \\
\hline & Coefficient & $95 \% \mathrm{Cl}$ & Coefficient & $95 \% \mathrm{Cl}$ & Coefficient & $95 \% \mathrm{Cl}$ \\
\hline \multicolumn{7}{|c|}{ Milk consumption categories ${ }^{\star *}$} \\
\hline No milk & Reference & & Reference & & Reference & \\
\hline$<250 \mathrm{~mL} / \mathrm{d}$ & -0.29 & -0.59 to 0.01 & -0.15 & -0.33 to 0.04 & -0.10 & -0.26 to 0.06 \\
\hline $250-500 \mathrm{~mL} / \mathrm{d}$ & $-0.39^{*}$ & -0.70 to -0.09 & $-0.20^{\star}$ & -0.39 to $0 .-02$ & -0.05 & -0.21 to 0.11 \\
\hline$>500 \mathrm{~mL} / \mathrm{d}$ & $-0.45^{*}$ & -0.82 to -0.07 & $-0.25^{\star}$ & -0.48 to -0.02 & -0.13 & -0.33 to 0.08 \\
\hline \multicolumn{7}{|c|}{$\begin{array}{l}\text { Total dairy consumption } \\
\text { categories } \dagger \dagger\end{array}$} \\
\hline$<200 \mathrm{~g} / \mathrm{d}$ & Reference & & Reference & & Reference & \\
\hline $200-399 \mathrm{~g} / \mathrm{d}$ & 0.06 & -0.26 to 0.15 & -0.10 & -0.22 to 0.03 & -0.08 & -0.19 to 0.02 \\
\hline $400-799 \mathrm{~g} / \mathrm{d}$ & -0.17 & -0.39 to 0.04 & -0.11 & -0.24 to 0.01 & -0.03 & -0.14 to 0.10 \\
\hline$\geq 800 \mathrm{~g} / \mathrm{d}$ & -0.04 & -0.44 to 0.35 & -0.15 & -0.39 to 0.09 & -0.05 & -0.27 to 0.18 \\
\hline
\end{tabular}

${ }^{*} \mathrm{P}<0.05$.

†Multivariable linear regression performed on baseline data (cross sectional) of 788 women aged $\geq 50$ years; serum marker of systemic inflammation (hsCRP) and bone turnover (CTx-bone resorption: P1NP-bone formation) are log transformed.

$\ddagger$ Model adjusted for BMI, mobility, diabetes, oral glucocorticoids, hypertension.

§Model adjusted for BMI, age, bisphosphonate, HT.

१Model adjusted for age, HT, diabetes.

**Milk comprises skim, low fat, full fat with a serving size of $1 \mathrm{cup}=250 \mathrm{~mL}$.

††Total dairy includes milk, cheese, yoghurt and ice cream.

$\mathrm{BMI}$, body mass index; CTx, C-terminal telopeptide; hsCRP, high-sensitivity C reactive protein; HT, hormonal replacement therapy; P1NP, procollagen type $1 \mathrm{~N}$-terminal propeptide.

Our study has several strengths. One strength of the study is that GOS comprises a randomly selected group of participants, which has shown to be similar to the broader Australian population (in terms of income, socioeconomic status, etc). However, our findings are not generalisable to men nor other countries with different distributions of ethnicities, diet patterns and other factors. However, our study results are likely generalisable to the broader Australian population of women. We were able to perform a longitudinal analysis that incorporated a long follow-up time with a median of 14.26 years as GOS is a cohort study. Additionally, data on the main exposure variable and confounders were updated several times during the period of follow-up, which enhanced the robustness of our analyses. Cognisant that osteoporosis is a multifactorial disease, we included many possible potential confounders (age, oral glucocorticoids, HT and past fractures) in the analysis. Also, we used an objective method of ascertaining/confirming incident fractures from radiological reports rather than relying on self-reported information. However, this was a regionally validated fracture ascertainment method, which may not account for fractures in participants who left the region (figure 1).

However, our study did have some limitations. We were unable to describe the association between milk/total dairy intake and fracture risk, using U-/J-shaped graphs to show higher risks in the zero and high consumption groups and the lowest risk in the low-intermediate consumption groups because of the low number of fractures; which in turn may lead to lower precision in the estimates. The study sample size was modest. A post hoc power calculation showed that based on annual fracture rate of 14.10 per 1000 in the reference group $(<250 \mathrm{~mL} / \mathrm{d}$ milk consumption) the minimum detectable effect size (ie, relative risk) ranged from 1.5 to 1.9 , which was bigger than observed risk ratios from unadjusted and adjusted Cox models. Although the dietary questionnaire was designed to provide information on participants' habitual dairy intake, it is possible that dairy contained in manufactured/prepared products is not captured and thereby it underestimates total dairy consumption. In addition, when querying about the type of milk consumed, A2 milk/milk products (which contains exclusively A2 milk proteins) were not provided as an option to be selected by the participant; thus, we were not able to investigate particular milk proteins as potential mediators in association with milk consumption. Also, total dairy consumption in the Cox regression analysis was not time updated as dietary information was not collected consistently across all follow-up visits and we thus performed an analysis using the baseline data only. This might have led to unaccounted changes in exposure status that may have occurred during the period of follow-up. In addition, some participants were lost to follow-up during the study due to leaving the region $(n=29)$, which prevented time-dependent updates on their information. As with all observational/follow-up studies, attrition is unavoidable. In the interim, there may have been other unrecognised confounding in our study. 


\section{CONCLUSION}

Taken together, our study results suggest that higher milk consumption is not associated with increased risk for MOF; however, zero milk consumption appears to be associated with an increased risk for MOF. Also, higher consumption of total dairy (milk, yoghurt, cheese and ice cream) may increase the risk for MOF, indicating a negative influence on bone health. Further studies are warranted to identify optimal levels of milk and total dairy consumption ranges and the potential mechanisms by which total dairy consumption may influence the risk for fracture.

\section{Author affiliations}

${ }^{1}$ School of Medicine, IMPACT SRC, Deakin University, Geelong, Victoria, Australia ${ }^{2}$ Faculty of Health,Biostatistics Unit, Deakin University, Burwood, Victoria, Australia ${ }^{3}$ Centre for Adolescent Health, Murdoch Children's Research Institute, Melbourne, Victoria, Australia

${ }^{4}$ Black Dog Institute, Sydney, New South Wales, Australia

${ }^{5}$ Department of Medicine, Western Campus, The University of Melbourne, St Albans, New South Wales, Australia

${ }^{6}$ Department of Epidemiology and Preventive Medicine, Monash University, Melbourne, Victoria, Australia

${ }^{7}$ University Hospital Geelong, Barwon Health, Geelong, Victoria, Australia

Acknowledgements The authors thank Professor Graham Giles of the Cancer Epidemiology Centre of The Cancer Council Victoria, for permission to use the Dietary Questionnaire for Epidemiological Studies (Version 2), Melbourne: The Cancer Council Victoria 1996 and Australian Institute for Health and Welfare for providing information on deaths.

Contributors HA, KH, MM, FNJ and JP contributed to the interpretation of data and critical appraisal of the manuscript, and HA constructed the manuscript.

Funding The Geelong 0steoporosis Study (GOS) was funded by the Victorian Health Promotion Foundation, and the National Health and Medical Research Council (NHMRC) Australia (projects 251638, 628582). The funding organisations played no role in the design or conduct of the study; in the collection, management analysis and interpretation of the data; nor in the preparation, review and approval of the manuscript.

Competing interests $\mathrm{HA}$ is supported by Deakin University Postgraduate Industry Research Scholarship, KH is supported by an Alfred Deakin Postdoctoral Research Fellowship and FNJ is supported by an NHMRC Career Development Fellowship (2) (1108125). The Food \& Mood Centre at the IMPACT SRC has received funding from the A2 Milk Company for an investigator-initiated randomised controlled trial (2018-2020).

\section{Patient consent for publication Not required.}

Ethics approval The study was approved by the Human Research Ethics Committee at Barwon Health.

Provenance and peer review Not commissioned; externally peer reviewed. Data availability statement Data are available upon reasonable request.

Open access This is an open access article distributed in accordance with the Creative Commons Attribution Non Commercial (CC BY-NC 4.0) license, which permits others to distribute, remix, adapt, build upon this work non-commercially, and license their derivative works on different terms, provided the original work is properly cited, appropriate credit is given, any changes made indicated, and the use is non-commercial. See: http://creativecommons.org/licenses/by-nc/4.0/.

\section{REFERENCES}

1 McCormick RK. Osteoporosis: integrating biomarkers and other diagnostic correlates into the management of bone fragility. Altern Med Rev 2007;12.

2 Bartolozzi E. The natural approach to osteoporosis. Clin Cases Miner Bone Metab 2015;12.
3 Pasco JA, Seeman E, Henry MJ, et al. The population burden of fractures originates in women with osteopenia, not osteoporosis. Osteoporos Int 2006;17:1404-9.

4 Shuler FD, Conjeski J, Kendall D, et al. Understanding the burden of osteoporosis and use of the world Health organization FRAX. Orthopedics 2012;35:798-805.

5 Rubenstein LZ. Falls in older people: epidemiology, risk factors and strategies for prevention. Age Ageing 2006;35 Suppl 2:ii37-41.

6 Thorell K, Ranstad K, Midlöv P, et al. Is use of fall risk-increasing drugs in an elderly population associated with an increased risk of hip fracture, after adjustment for multimorbidity level: a cohort study. BMC Geriatr 2014:14:131.

7 Delbaere K, Close JCT, Heim J, et al. A multifactorial approach to understanding fall risk in older people. J Am Geriatr Soc 2010;58:1679-85.

8 Masud T, Frost M, Ryg J, et al. Central nervous system medications and falls risk in men aged $60-75$ years: the study on male osteoporosis and aging (soma). Age Ageing 2013;42:121-4.

9 Recker RR, Deng H-W. Role of genetics in osteoporosis. Endocrine 2002;17:55-66.

10 Duncan EL, Danoy P, Kemp JP, et al. Genome-Wide association study using extreme truncate selection identifies novel genes affecting bone mineral density and fracture risk. PLoS Genet 2011; 7:e1001372.

11 Rizzoli R, Bonjour J, Osteoporosis FS. Genetics and hormones. J Mol Endocrinol 2001;26:79-94.

12 Henry MJ, Pasco JA, Nicholson GC, et al. Prevalence of osteoporosis in Australian women: Geelong osteoporosis study. $J$ Clin Densitom 2000;3:261-8.

13 Alswat KA. Gender disparities in osteoporosis. J Clin Med Res 2017;9:382-7.

14 Heaney RP, Calcium HRP. Calcium, dairy products and osteoporosis. J Am Coll Nutr 2000;19:83S-99.

15 Pasco JA, Henry MJ, Nicholson GC, et al. Behavioural and physical characteristics associated with vitamin D status in women. Bone 2009;44:1085-91.

16 Rizzoli R, Stevenson JC, Bauer JM, et al. The role of dietary protein and vitamin $\mathrm{D}$ in maintaining musculoskeletal health in postmenopausal women: a consensus statement from the European Society for clinical and economic aspects of osteoporosis and osteoarthritis (ESCEO). Maturitas 2014;79:122-32.10.1016/j. maturitas.2014.07.005

17 Flynn A. The role of dietary calcium in bone health. Proc. Nutr. Soc. 2003:62:851-8

18 Harel Z, Riggs S, Vaz R, et al. Adolescents and calcium: what they do and do not know and how much they consume. Journal of Adolescent Health 1998;22:225-8.

19 Pasco JA, Sanders KM, Henry MJ, et al. Calcium intakes among Australian women: Geelong osteoporosis study. Aust N Z J Med 2000;30:21-7.

20 International Osteoporosis Foundation Fact sheet. Milk and dairy products are good for bone health 2015.

21 Ebeling PR, Eisman J. Recommendations from the vitamin D and calcium forum 2005.

22 Michaelsson K, Wolk A, Langenskiold S, et al. Milk intake and risk of mortality and fractures in women and men: cohort studies. BMJ 2014;349:96015.

23 Feskanich D, Meyer HE, Fung TT, et al. Milk and other dairy foods and risk of hip fracture in men and women. Osteoporos Int 2018;29:385-96.

24 Holvik K, Meyer HE, Laake I, et al. Milk drinking and risk of hip fracture. The Norwegian epidemiologic osteoporosis studies (NOREPOS). Br J Nutr 2018:1-21.

25 Bian S, Hu J, Zhang K, et al. Dairy product consumption and risk of hip fracture: a systematic review and meta-analysis. BMC Public Health 2018;18:165.

26 Cui X, Zuo P, Zhang Q, et al. Chronic systemicD-galactose exposure induces memory loss, neurodegeneration, and oxidative damage in mice: protective effects of R- $\alpha$-lipoic acid. J Neurosci Res 2006;84:647-54.

27 Trivedi MS, Hodgson NW, Walker SJ, et al. Epigenetic effects of casein-derived opioid peptides in SH-SY5Y human neuroblastoma cells. Nutr Metab 2015;12.

$28 \mathrm{MRU} H$, Kapila R, Saliganti V. Consumption of $\beta$-casomorphins-7/5 induce inflammatory immune response in mice gut through th 2 pathway. J Funct Foods 2014;8:150-60.

29 Sendur OF, Turan Y, Tastaban E, et al. Antioxidant status in patients with osteoporosis: a controlled study. Joint Bone Spine 2009;76:514-8

30 Clowes JA, Riggs BL, Khosla S. The role of the immune system in the pathophysiology of osteoporosis. Immunol Rev 2005;208:207-27. 
31 Pasco JA, Williams LJ, Brennan-Olsen SL, et al. Milk consumption and the risk for incident major depressive disorder. Psychother Psychosom 2015;84:384-6.

32 Iolascon G, Cervone M, Gimigliano R, et al. Neuropsychiatric disorders in hip fracture. Clin Cases Miner Bone Metab 2011;8.

33 Williams LJ, Pasco JA, Jackson $\mathrm{H}$, et al. Depression as a risk factor for fracture in women: a 10 year longitudinal study. J Affect Disord 2016;192:34-40.

34 Biver E, Durosier-lzart C, Merminod F, et al. Fermented dairy products consumption is associated with attenuated cortical bone loss independently of total calcium, protein, and energy intakes in healthy postmenopausal women. Osteoporos Int 2017:1-12.

35 McCabe L, Britton RA, Parameswaran N. Prebiotic and probiotic regulation of bone health: role of the intestine and its microbiome. Curr Osteoporos Rep 2015;13:363-71.

36 Pasco JA, Nicholson GC, Kotowicz MA. Cohort profile: Geelong osteoporosis study. Int J Epidemiol 2012;41:1565-75.

37 Pasco JA, Henry MJ, Gaudry TM, et al. Identification of incident fractures: the Geelong osteoporosis study. Aust N Z J Med 1999;29:203-6.

38 Pasco JA, Lane SE, Brennan-Olsen SL, et al. The epidemiology of incident fracture from cradle to senescence. Calcif Tissue Int 2015:97:568-76.

39 Holloway-Kew KL, Zhang Y, Betson A, et al. How well do the FRAX (Australia) and Garvan calculators predict incident fractures? data from the Geelong osteoporosis study. Osteoporos Int 2019:1-11.

40 Fracture risk assessment tool. Available: https://wwwsheffieldacuk/FRAX

41 Hodge A, Patterson AJ, Brown WJ, et al. The anti cancer Council of Victoria FFQ: relative validity of nutrient intakes compared with weighed food records in young to middle-aged women in a study of iron supplementation. Aust N Z J Public Health 2000;24:576-83.

42 Australian Bureau of Statistics. Census of Population and Housing: Socio-Economic Indexes for Areas 2016(2033.0.55.001).

43 Jenkins N, Black M, Paul E, et al. Age-Related reference intervals for bone turnover markers from an Australian reference population. Bone 2013;55:271-6.

44 Pasco JA, Kotowicz MA, Henry MJ, et al. High-Sensitivity C-reactive protein and fracture risk in elderly women. JAMA 2006;296:1349-55.

45 Delaney MF. Strategies for the prevention and treatment of osteoporosis during early postmenopause. Am J Obstet Gynecol 2006;194:S12-23.

46 Murphy S, Khaw K-T, May H, et al. Milk consumption and bone mineral density in middle aged and elderly women. BMJ 1994;308:939-41.
47 Huncharek M, Muscat J, Kupelnick B. Impact of dairy products and dietary calcium on bone-mineral content in children: results of a meta-analysis. Bone 2008;43:312-21.

48 Tang BMP, Eslick GD, Nowson C, et al. Use of calcium or calcium in combination with vitamin D supplementation to prevent fractures and bone loss in people aged 50 years and older: a meta-analysis. The Lancet 2007;370:657-66.

49 Song X, Bao M, Li D, et al. Advanced glycation in Dgalactose induced mouse aging model. Mech Ageing Dev 1999;108:239-51.

50 MRU H, Kapila R, Shandilya UK, et al. Impact of milk derived $\beta$-casomorphins on physiological functions and trends in research: a review. Int J Food Prop 2014;17:1726-41.

51 Laugesen M, Elliott R. Ischaemic heart disease, type 1 diabetes, and cow milk A1 $\beta$-casein. N Z Med J 2003;116.

52 Tailford K, Berry CL, Thomas AC. A casein variant in cow's milk is atherogenic. Atherosclerosis 2003;170:13-19.

53 Bell SJ, Grochoski GT, Clarke AJ. Health implications of milk containing beta-casein with the A2 genetic variant. Crit Rev Food Sci Nutr 2006;46:93-100.

54 Birgisdottir BE, Hill JP, Thorsson AV, et al. Lower consumption of cow milk protein $A 1 \beta$-casein at 2 years of age, rather than consumption among 11- to 14-year-old adolescents, may explain the lower incidence of type 1 diabetes in Iceland than in Scandinavia. Ann Nutr Metab 2006;50:177-83.

55 Elliott RB, Harris DP, Hill JP, et al. Type I (insulin-dependent) diabetes mellitus and cow milk: casein variant consumption. Diabetologia 1999;42:292-6.

56 Bischoff-Ferrari HA, Dawson-Hughes B, Baron JA, et al. Milk intake and risk of hip fracture in men and women: a meta-analysis of prospective cohort studies. J Bone Miner Res 2011;26:833-9.

57 Hardy R, Cooper MS. Bone loss in inflammatory disorders. J Endocrinol 2009;201:309-20.

58 Ginaldi L, Di Benedetto M, De Martinis M. Inflammation and ageing. Immun Ageing 2005;2.

59 Eriksson AL, Movérare-Skrtic S, Ljunggren Östen, et al. HighSensitivity CRP is an independent risk factor for all fractures and vertebral fractures in elderly men: the MROS Sweden study. $J$ Bone Miner Res 2014;29:418-23.

60 Panagiotakos DB, Pitsavos $\mathrm{CH}$, Zampelas AD, et al. Dairy products consumption is associated with decreased levels of inflammatory markers related to cardiovascular disease in apparently healthy adults: the Attica study. J Am Coll Nutr 2010;29:357-64. 